\title{
Change Detection Algorithm Based on U-NET Convolutional Neural Network for Multitemporal Wavelength-Resolution SAR Images
}

\author{
Rodrigo Aragão Santos, Marcelo da Silva Pinho, and Renato Machado
}

\begin{abstract}
Este artigo apresenta um algoritmo de detecção de mudança (CDA - change detection algorithm) para imagens de radar de abertura sintética (SAR) de resolução de comprimento de onda multitemporais baseado na arquitetura de redes neurais convolucionais (CNN - convolutional neural network) do tipo U-NET. O algoritmo considera filtragem não linear para extrair informação de texturas de imagens SAR de amplitude adquiridas pelo sistema sueco CARABAS-II. Como as detecções de mudanças ocorrem em imagens multitemporais, as texturas relevantes (entropia e variância) podem ser obtidas a partir dos histogramas das imagens SAR resultantes de operações de soma e diferença de imagens. As imagens originais e as texturas obtidas são dadas como entrada do algoritmo proposto. Como resultado, o CDA proposto apresenta desempenho de detecção semelhante ao de outros métodos, mas o número de falsos alarmes é significativamente reduzido.
\end{abstract}

Abstract-This paper presents a change detection algorithm (CDA) for multitemporal wavelength-resolution synthetic aperture radar (SAR) images based on U-NET convolutional neural network (CNN) architecture. The algorithm uses non-linear filtering to extract textural information from amplitude SAR images. Since we want to detect changes in multitemporal acquisitions, releva nt textures (entropy and variance) can be obtained from the histograms of sum and difference SAR images. The original images and the obtained textures are given as input of the proposed algorithm. As a result, the proposed CDA has a similar detection performance compared to other methods, but the number of false alarms is significantly reduced.

Keywords-CDA, CNN, SAR Images, Textures, Machine Learning, Signal Processing.

\section{INTRODUCTION}

Synthetic Aperture Radar (SAR) technology is extensively used for remote sensing of the Earth, providing high-resolution SAR images for many different applications, such as climate and environment monitoring and target detection [1], [2].

In multi-temporal acquisitions, we can either access the information contained in the entire time frame or detect what has changed between acquisitions. When the latter is the focus, change detection algorithms (CDA) are traditionally used to identify the relevant changes [3], [4].

Change detection algorithms aim to provide a high percentage of true positives with as low as possible false positives. Those can be relevant in many applications, such as detecting

Rodrigo Aragão, Instituto Tecnológico de Aeronáutica, São José dos Campos-SP, e-mail: rodrigo.aragao.santos@gmail.com; Marcelo da S. Pinho, Instituto Tecnológico de Aeronáutica, São José dos Campos-SP, e-mail: mpinho@ita.br. Renato Machado, Instituto Tecnológico de Aeronáutica, São José dos Campos-SP, e-mail: rmachado@ita.br. hidden objects over a large area, where a small number of false positives is vital in the quality of the final data for operation application. Before the popularization of Machine Learning algorithms, CDAs were mainly based on traditional statistical decision theory, e.g., traditional hypothesis testing criterion methods, such as maximum a posteriori criterion [6], likelihood ratio test [7], [8], [9], generalized likelihood ratio test [10], [11], [12], or Bayesian theory approaches [13], [14]. Several of those CDAs have achieved high accuracy in terms of true positives, but most show unsatisfactory performance in terms of false positives percentage [3], [4], [18], [19].

In the last few years, aiming at overcoming this limitation of traditional CDA approaches, many studies have been developing considering the use of machine learning algorithms for target and change detection applications [15], [16]. Basically, there are two types of machine learning algorithms: supervised and unsupervised. They differ mainly on whether a classification reference is used to guide the algorithm for the classification of the targets [5]. When working with machine learning algorithms, it is important to define the relevant parameters to assess performance. Mainly, they are a subset of the number of true positives, false positives, true negatives, and false negatives [17]. As those quantities are usually not independent, their prioritization comes with trade-offs, so it is keen to establish which one better fits the nature of the problem. For instance, when dealing with forest monitoring, one is typically concerned with the number of true positive detections (where each positive detection would represent a tree or forest pixel) and the number of false positives [3]. We consider these metrics to assess the performance of the proposed algorithm.

We propose a CDA based on U-NET CNN for detecting cars hidden under tree foliage. A similar change detection approach was presented in [21] to conventional SAR images, i.e., for images acquired for higher frequencies (microwave systems). Aiming to reduce the number of false positives, we propose using textural information to improve these methods as done in [20], keeping in mind the trade-off between the number of false positives and the number of true positives. Throughout statistical processing methods, local information (textural information) can be extracted and selected as additional information for machine learning algorithms. Moreover, since CNNs use convolutions, which are based on linear filters, to achieve better classifications, we do not use linear textural information as input to the CNNs since they could be redundant. When comparing the proposed technique with other 
algorithms on the same dataset, we show that the results show similar detection performance but presenting a significant false alarm rate improvement.

The remainder of the paper is organized as follows. Section II presents the basics of textural feature extraction. Section III presents the proposed method and discusses its advantages against false alarms. Section IV presents the dataset and the proposed algorithm. Section V provides the results and some discussions. Finally, Section VI gives some conclusions and final remarks.

\section{TEXTURE INFORMATION EXTRACTION}

Texture Information [22], [23], [24] has been demonstrated several times to provide important information about the spatial dependency of neighboring pixels when interpreting SAR images [25], [20]. Throughout this paper, textural information is extracted from the backscatter content present in the SAR images, and it is used as additional input to the machine learning algorithm. There are several methods for extracting textural information. In this paper, we focus on the Sum and Difference Histogram (SADH) method [25].

For a given region in a SAR image, we consider that the amplitude of the backscatter can be modeled as the realization of a bi-dimensional stationary processes represented by the random variables $u_{[x, y]}$. The first step is to quantize the random variable $u$ to have $N_{g}$ discrete possible values. The second step is to obtain the joint probability distribution function of the random vector $\left[u_{[x, y]}, u_{[x+\delta x, y+\delta y]}\right]$ which is given by a $N_{g} \times N_{g}$ bi-dimensional matrix. This matrix is called Gray Level Co-Occurrence Matrix (GLCM) [22]. Notice that the GLCM matrix contains the information about the relationship of two neighboring pixels, identified by the displacement vector $\delta=(\delta x, \delta y)$. Furthermore, since the GLCM is the direct result of comparing two neighboring pixels, it then describes the spatial relationship between them, i.e., the pattern that appears between two neighboring pixels throughout the image.

Textural information is then obtained directly from the GLCM through transformations such as, sum of elements, trace of the matrix, Frobenius norm of the matrix, etc. A drawback of this method is the computational time needed to obtain the GLCM. The time complexity to obtain the GLCM matrix is $O\left(N_{g}^{2}\right)$, which can grow fast when the number of gray levels is increased, therefore it is not a reasonable algorithm for real world applications.

To overcome this problem, we can use the SADH method. This method makes an estimation of the GLCM matrix, as presented in [24]. The SADH method assumes that the vector $\left[u_{[x, y]}, u_{[x+\delta x, y+\delta y]}\right]$ is modeled as a random Gaussian process, and therefore a linear transformation can be applied to this vector to create another random Gaussian vector, which has uncorrelated components. The new vector is $\left[s_{[x, y]}, d_{[x, y]}\right]$ and is obtained as follows

$$
\begin{aligned}
& s_{[x, y]}=u_{[x, y]}+u_{[x+\delta x, y+\delta y]} \\
& d_{[x, y]}=u_{[x, y]}-u_{[x+\delta x, y+\delta y]}
\end{aligned}
$$

By taking advantage of the fact that $\left[s_{[x, y]}, d[x, y]\right]$ has uncorrelated Gaussian and, therefore, independent variables, we can estimate the joint probability distribution of the original random vector $\left[u_{[x, y]}, u_{[x+\delta x, y+\delta y]}\right]$ as follows

$$
\begin{array}{r}
P\left(u_{[x, y]}=u_{1}, u_{[x+\delta x, y+\delta y]}=u_{2}\right)= \\
P\left(s_{[x, y]}=u_{1}+u_{2}, d_{[x, y]}=u_{1}-u_{2}\right)= \\
P\left(s_{[x, y]}=u_{1}+u_{2}\right) \cdot P\left(d_{[x, y]}=u_{1}-u_{2}\right)
\end{array}
$$

With this formulation, the joint distribution is fully encoded in the two 1-dimensional probability distributions $P\left(s_{[x, y]}\right)$ and $P\left(d_{[x, y]}\right)$. The original random vector $\left[u_{[x, y]}, u_{[x+\delta x, y+\delta y]}\right]$ may not be Gaussian, since this is only an approximation. However, for most applications, SAR images predominantly contain homogeneous areas, such as lakes and forests, which can be well-approximated to a Gaussian distribution. The advantage of the SADH technique arises from the fact that the probability density functions $P\left(s_{[x, y]}\right)$ and $P\left(d_{[x, y]}\right)$ are one-dimensional arrays with $N_{g}$ elements each, i.e., the time complexity is reduced from $O\left(N_{g}^{2}\right)$ to $O\left(2 N_{g}\right)$, that is, from a quadratic to a linear time complexity, making the process adequate for real-world applications.

After obtaining the probability density functions $P\left(s_{[x, y]}\right)$ and $P\left(d_{[x, y]}\right)$, a set of textural information can be extracted as follows (for better readability, we define $P_{s}(i)=P(s[x, y]=$ i) and $\left.P_{d}(j)=P(d[x, y]=j)\right)$ :

1) Mean $(\mu)$ : describes the mean value of the cooccurrences and is given by

$$
\text { MEAN }=\frac{1}{2} \sum_{i=1}^{N_{g}} i \cdot P_{s}(i)
$$

2) Cluster prominence: describes the fourth moment of the random variables and is given by

$$
\mathrm{CLP}=\sum_{i=1}^{N_{g}}(i-2 \mu)^{4} \cdot P_{s}(i)
$$

3) Cluster shade: describes the third moment of the random variables and is given by

$$
\mathrm{CLS}=\sum_{i=1}^{N_{g}}(i-2 \mu)^{3} \cdot P_{s}(i)
$$

4) Contrast: measure the difference of intensities of cooccurrences in the image and is given by

$$
\mathrm{CON}=\sum_{j=\frac{-N_{g}}{2}}^{\frac{N_{g}}{2}}(j)^{\cdot} P_{d}(j)
$$

5) Correlation: describes the linear correlation of intensities of co-occurrences in the image and is given by

$$
\mathrm{COR}=\frac{1}{2} \sum_{i=1}^{N_{g}}(i-2 \mu)^{2} \cdot P_{s}(i)-\frac{1}{2} \sum_{j=\frac{-N_{g}}{2}}^{\frac{N_{g}}{2}}(j) \cdot P_{d}(j)
$$

6) Energy: describes the energy of the received signal and is given by

$$
\mathrm{ENE}=\sum_{i=1}^{N_{g}} P_{s}(i)^{2} \cdot \sum_{j=\frac{-N_{g}}{2}}^{\frac{N_{g}}{2}} P_{d}(j)^{2}
$$


7) Entropy: describes the uncertainty of the co-occurrence matrix and is given by

$$
\mathrm{ENT}=-\sum_{i=1}^{N_{g}} P_{s}(i) \log \left(P_{s}(i)\right)-\sum_{j=\frac{-N_{g}}{2}}^{\frac{N_{g}}{2}} P_{d}(j) \log \left(P_{d}(j)\right)
$$

8) Homogeneity: describes the degree of similarity between images and is given by

$$
\mathrm{HOM}=-\sum_{j=\frac{-N_{g}}{2}}^{\frac{N_{g}}{2}} \frac{P_{d}(j)}{1+j^{2}}
$$

9) Variance: describes the degree of dispersion of intensities between two images and is given by

$$
\mathrm{VAR}=\frac{1}{2} \sum_{i=1}^{N_{g}}(i-2 \mu)^{2} P_{s}(i)+\frac{1}{2} \sum_{j=\frac{-N_{g}}{2}}^{\frac{N_{g}}{2}} j^{2} P_{d}(j)
$$

\section{MAterials AND Methods}

CARABAS-II is a SAR system, operating in the VHF band (20-90 MHz), in $\mathrm{HH}$ polarization, and is the second generation SAR system from the Swedish CARABAS radar. The system was used to acquire SAR images over a forest area in northern Sweden during a campaign in 2002. The set of 24 images was made publicly available for the scientific community after being preprocessed, i.e., geocoded, calibrated, equalized, and co-registered, before the dataset was made available [3]. It is worth mentioning that all phase information was removed from the dataset, remaining only amplitude information, so interferometric and coherence techniques cannot be executed on this dataset.

Each magnitude SAR image covers an area of $2 \times 3 \mathrm{~km}^{2}$. For the CARABAS-II system, the resolution in range and azimuth is approximately $2.5 \mathrm{~m}$, as reported in [3], and the final pixel resolution is $1 \mathrm{~m}$. Therefore, each image consists of 6 million pixels, organized in a matrix of 3000 rows by 2000 columns. In each image, 25 military vehicles were hidden under the tree foliage. As mentioned before, detecting those military vehicles while trying to lower the number of false alarms is the goal of this study. In Figure 1, it is shown an example of image acquired with the CARABAS-II SAR system.

The targets, i.e., military vehicles, are not all of the same size. There are 10 TGB 11 , with dimensions $4.4 \times 1.9 \times 2.2 \mathrm{~m}, 8$ TGB30, with dimensions $6.8 \times 2.5 \times 3.0 \mathrm{~m}$ and 7 TGB40, with dimensions $7.8 \times 2.5 \times 3.0 \mathrm{~m}$. Also, the images were acquired with different flight headings. There were two missions with a flight heading of 225 degrees, two with a flight heading of 135 degrees and two with a flight heading of 0 degrees. The reference for the flight heading is 0 degree toward north, increasing clockwise.

\section{Proposed Algorithm for Change Detection}

In [21], it is presented a UNET-based change detection algorithm that relies on classifying the differences between two acquisitions. We chose to use this idea in our method

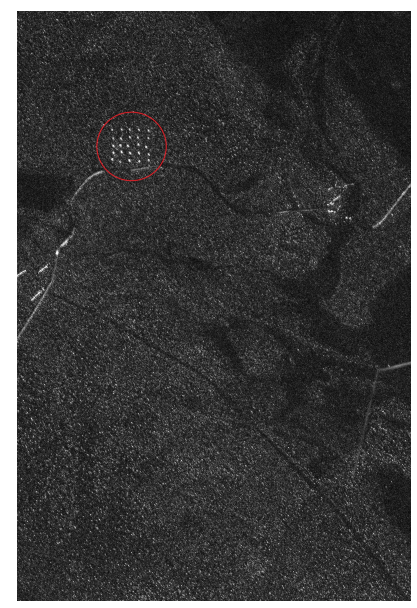

Fig. 1. Example of a SAR image acquired by the CARABAS-II system. The red circle shows the position of the cars hidden under a canopy of trees.

with some modifications to better suit our change detection problem. In [21], the algorithm can detect changes when there are multiple different classes in the input images. In this paper, there are only two classes of interest: forest and cars hidden under the foliage. Considering only two classes makes the method more robust against false alarms when the input image has only two classes.

The first step of the algorithm is to calculate the textures for each SAR image. Multiple textures can be generated and used as information for machine learning algorithms. However, we selected only two textures - entropy and variance - to use as additional information to the algorithm. These two textures were chosen based on the fact that linearly dependent textures will be naturally fitted by the convolution filters of the CNNs, and thus will not provide much additional information. Moreover, we have decided to use only two textures since we do not want to increase too much the total computational cost for the algorithm.

Figures 2 and 3 show the SAR images after obtaining the chosen textures, i.e., the variance and entropy.

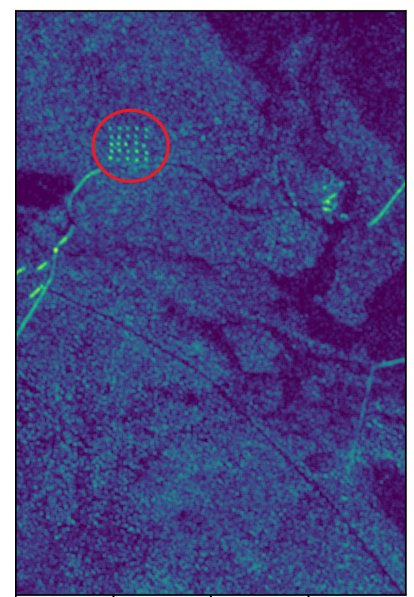

Fig. 2. Entropy image. The red circle shows the position of the vehicles.

Next, the textures are combined with the original image to create a three-channel tensor, which is also used as input of 


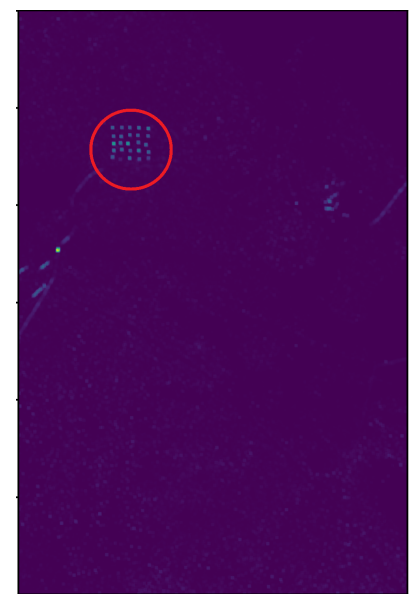

Fig. 3. Variance image. The red circle shows the position of the vehicles.

UNET convolutional neural network. After creating 24 image tensors, the dataset was divided into 12 tensors for training purposes and other 12 tensors for validation purposes. The first 12 tensors from the first 12 images were used for training purposes and the last 12 were used to validation purposes.

After performing the training of the UNET network we use these trained UNETs as part of the change detection algorithm. The CDA work as follows: in order to detect what has changed between two images there is a 4 step process:

1) Feed the first tensor as a input to the UNET and get the classification

2) Feed the second tensor as input to the UNET, and after that alternate the classes from the final image (switch all car classification pixels by forest classification pixels and vice-versa)

3) Take the absolute value of the difference of the tensors, perform a bias correction (the value for the bias correction was adjusted manually). After that feed this tensor as input to the UNET.

4) Get the three classifications obtained prior and take the intersection of all classes (in order to a pixel to be classified as a car in the final image, it has to be a car in all of the three images at the same time)

The intuition of the algorithm works as follows: In order to a car be detected as change in the image number 1 then it must be able to be detected by itself on the image 1 . In order to a car to have appeared in image 1 then it must not be in the image 2 , by alternating the classes the algorithm eliminates cars that are detected in image 2 but did not change position. The final step of the algorithm is to classify the difference between the images, this is the idea originally proposed by ([21]) and it is also added to the algorithm as a way to make the algorithm more robust against false detections.

After the final classification image is created by the CDA, it was still performed a threshold filter selection to eliminate pixel classification noise that was created by the algorithm it was considered only sets of pixel which had 100 or more connected components.

\section{RESUlTS}

As previously mentioned, the dataset was divided into two halves: 12 first twelve images were used to train the UNET $\mathrm{CNN}$ and the last 12 were used as validation purposes. It was selected 24 pairs of images to assess the quality of the change detection algorithm - the 24 pairs selected followed the standard selection used for CDA testing and validation of the CARABAS-II dataset as it was done in ([3], [4], [18]).

The results are shown in Table I, where each image M_P was labelled in terms of Mission number (M) and flight pass (P). The compared images have different mission numbers, but the same flight pass heading. In Table I is presented the results of the CDA without the textural information to demonstrate the how the textures contribute as additional information to machine learning algorithms.

\begin{tabular}{|c|c|c|c|c|c|}
\hline $\begin{array}{l}\text { Monitored } \\
\text { Image }\end{array}$ & $\begin{array}{l}\text { Reference } \\
\text { Image }\end{array}$ & $\begin{array}{l}\text { Detected } \\
\text { Targets } \\
\text { (with } \\
\text { texture) }\end{array}$ & $\begin{array}{l}\text { False } \\
\text { Alarms } \\
\text { (with } \\
\text { texture) }\end{array}$ & $\begin{array}{l}\text { Detected } \\
\text { Targets } \\
\text { (without } \\
\text { texture) }\end{array}$ & $\begin{array}{l}\text { False } \\
\text { Alarms } \\
\text { (without } \\
\text { texture) }\end{array}$ \\
\hline $2 \_1$ & 3_1 & 25 & 0 & 25 & 0 \\
\hline 3_1 & 4_1 & 23 & 1 & 21 & 1 \\
\hline 4_1 & 5_1 & 25 & 0 & 25 & 0 \\
\hline 5_1 & 2_1 & 25 & 0 & 25 & 0 \\
\hline $2 \_2$ & $4 \_2$ & 25 & 0 & 25 & 0 \\
\hline 3_2 & $5 \_2$ & 25 & 0 & 23 & 1 \\
\hline 4_2 & $2 \_2$ & 23 & 0 & 25 & 0 \\
\hline 5_2 & $3 \_2$ & 22 & 0 & 24 & 0 \\
\hline $2 \_3$ & $5 \_3$ & 25 & 0 & 25 & 0 \\
\hline 3_3 & $2 \_3$ & 22 & 1 & 21 & 1 \\
\hline 4_3 & 3_3 & 25 & 1 & 25 & 1 \\
\hline 5_3 & 4_3 & 25 & 0 & 25 & 0 \\
\hline $2 \_4$ & $3 \_4$ & 25 & 0 & 25 & 0 \\
\hline $3 \_4$ & 4_4 & 24 & 0 & 23 & 1 \\
\hline 4_4 & $5 \_4$ & 25 & 0 & 25 & 0 \\
\hline 5_4 & $2 \_4$ & 24 & 0 & 25 & 0 \\
\hline $2 \_5$ & $4 \_5$ & 25 & 0 & 25 & 0 \\
\hline $3 \_5$ & $5 \_5$ & 24 & 0 & 21 & 2 \\
\hline $4 \_5$ & $2 \_5$ & 25 & 0 & 25 & 0 \\
\hline 5_5 & $3 \_5$ & 23 & 0 & 24 & 0 \\
\hline $2 \_6$ & 5_6 & 25 & 0 & 25 & 0 \\
\hline $3 \_6$ & $2 \_6$ & 23 & 1 & 25 & 0 \\
\hline $4 \_6$ & $3 \_6$ & 24 & 0 & 25 & 0 \\
\hline 5_6 & $4 \_6$ & 25 & 1 & 25 & 0 \\
\hline
\end{tabular}

TABLE I

RESULTS OF CDA IN TERMS OF DETECTED TARGETS AND FALSE ALARM RATE FOR THE SAME IMAGE PAIRS CONSIDERED IN ([3]). IT IS ALSO PRESENTED THE RESULTS WHEN TEXTURAL INFORMATION IS EITHER USED OR NOT USED AS INPUT OF THE PROPOSED ALGORITHM.

The performance of the algorithm is measured in terms of probability detection $\left(P_{d}\right)$ which is the number of detected targets divided by the total targets, and the false alarm rate (FAR), which is the number of false detections per kilometer square. Using this algorithm, we achieved a probability of detection of $97 \%$ and a false alarm rate of $0.034 / \mathrm{km}^{2}$, while other algorithms such as [3] presented a $P_{d}$ of $97 \%$ and a $\mathrm{FAR}=0.67 / \mathrm{km}^{2}$. More recent algorithms such as [4], achieved a $P_{d}$ of around $97 \%$ and a FAR $=0.28 / \mathrm{km}^{2},[16]$ achieved a $P_{d}$ of about $97 \%$ and a FAR $=0.0313 / \mathrm{km}^{2}$, and [15] achieved a $P_{d}$ of around $99 \%$ and a FAR $=0.0833 / \mathrm{km}^{2}$, respectively. It worth mentioning that methods presented in [16], [15] are based on more complex CNN with greater time complexity. 


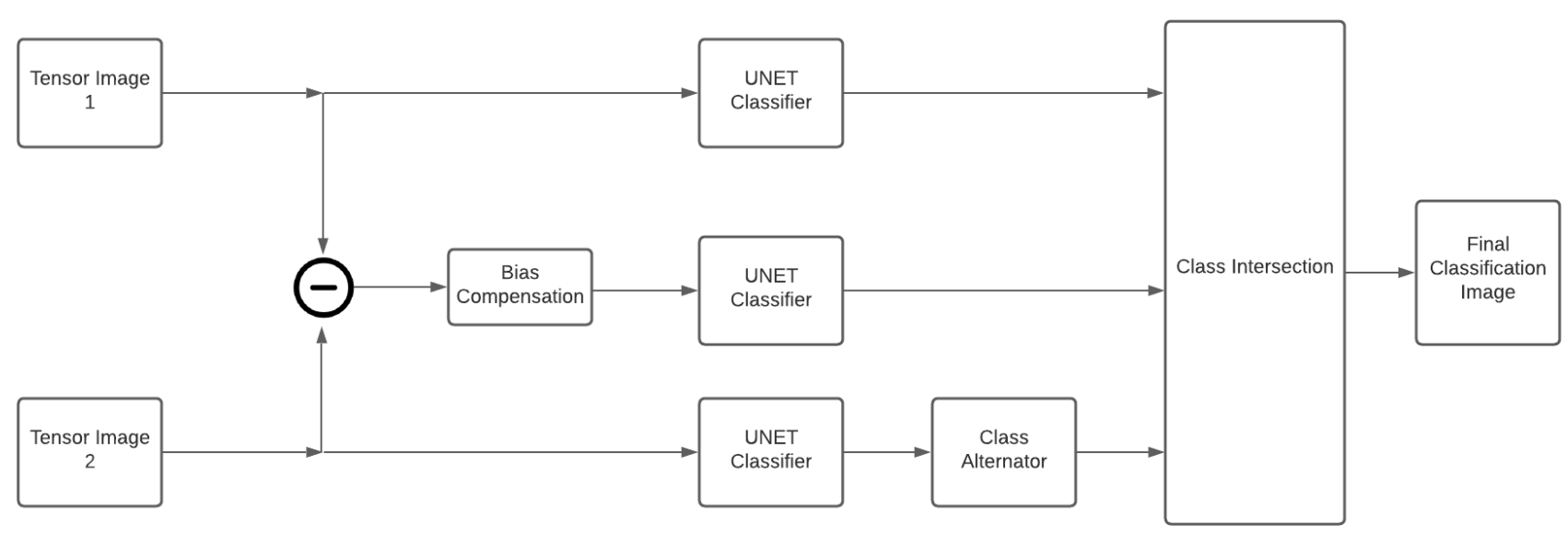

Fig. 4. Diagram of the proposed algorithm.

\section{CONCLUSION}

A texture based machine learning algorithm for change detection was proposed. The proposed algorithm showed similar performance in terms of probability of detection (97\%) in comparison to other CDAs. However, the proposed algorithm showed excellent performance in terms of false alarm rate (0.034), with a UNET based CDA with linear time complexity. Another approach presented a such small false alarm rate, however only the proposed method has linear time complexity.

\section{REFERENCES}

[1] A. Moreira, P. Prats-Iraola, M. Younis, G. Krieger, I. Hajnsek and K. P. Papathanassiou, "A tutorial on synthetic aperture radar," IEEE Geoscience and Remote Sensing Magazine, vol. 01, no. 01, pp. 6-43, March 2013.

[2] L. M. H. Ulander, H. Hellsten and G. Stenstrom, "Synthetic-aperture radar processing using fast factorized back-projection," IEEE Transactions on Aerospace and Electronic Systems, vol. 39, no. 03, pp. 760-776, July 2003.

[3] M. Lundberg, L. M. H. Ulander, W. E. Pierson, and A. Gustavsson, "A challenge problem for detection of targets in foliage," Proceedings of SPIE, 2006

[4] R. D. Molin, R. A. S. Rosa, F. M. Bayer, M. I. Pettersson and R. Machado, "A Change Detection Algorithm for Sar Images Based on Logistic Regression," IEEE International Geoscience and Remote Sensing Symposium (IGARSS), 2019, pp. 1514-1517.

[5] M. Alloghani, D. Al-Jumeily, J. Mustafina, A. Hussain, A. J. Aljaaf, "A Systematic Review on Supervised and Unsupervised Machine Learning Algorithms for Data Science," Springer, book series, pp. 3-21.

[6] S.M.Kay, "Fundamentals of Statistical Signal Processing, Detection Theory", 1st ed. New Jersey, USA: Prentice-Hall 1989.

[7] M. I. Pettersson, V. T. Vu, N. R. Gomes, P. Dammert and H. Hellsten, "Incoherent detection of man-made objects obscured by foliage in forest area," IEEE International Geoscience and Remote Sensing Symposium (IGARSS), 2017, pp. 1892-1895.

[8] V. T. Vu, "Wavelength-Resolution SAR Incoherent Change Detection Based on Image Stack," IEEE Geoscience and Remote Sensing Letters, vol. 14, no. 07, pp. 1012-1016, July 2017.

[9] V. T. Vu, N. R. Gomes, M. I. Pettersson, P. Dammert and H. Hellsten, "Bivariate Gamma Distribution for Wavelength-Resolution SAR Change Detection," IEEE Transactions on Geoscience and Remote Sensing, vol. 57, no. 01, pp. 473-481, Jan. 2019.

[10] H. Zhuang, Z. Tan, K. Deng and G. Yao, "Adaptive Generalized Likelihood Ratio Test for Change Detection in SAR Images," IEEE Geoscience and Remote Sensing Letters, vol. 17, no. 3, pp. 416-420, March 2020
[11] J. Barber, "A Generalized Likelihood Ratio Test for Coherent Change Detection in Polarimetric SAR," IEEE Geoscience and Remote Sensing Letters, vol. 12, no. 09, pp. 1873-1877, Sept. 2015.

[12] Weiying Zhao, S. Lobry, H. Maitre, J.-M. Nicolas and F. Tupin, "Urban area change detection based on generalized likelihood ratio test," 9th International Workshop on the Analysis of Multitemporal Remote Sensing Images (MultiTemp), 2017, pp. 1-4.

[13] D. I. Alves, B. G. Palm, H. Hellsten, V. T. Vu, M. I. Pettersson, R. Machado, B. F. Uchôa-Filho, "Wavelength-Resolution SAR Change Detection Using Bayes' Theorem," IEEE Journal of Selected Topics in Applied Earth Observations and Remote Sensing, vol. 13, pp. 55605568, 2020.

[14] H. Zhang, W. Ni, W. Yan, H. Bian, and J. Wu, "Fast SAR Image Change Detection Using Bayesian Approach Based Difference Image and Modified Statistical Region Merging," The Scientific World Journal, vol. 2014, Article ID 862875, 12 pages.

[15] J. G. Vinholi, D. Silva, R. Machado, and M. I. Pettersson, "CNN-Based Change Detection Algorithm for Wavelength Resolution SAR Images," IEEE Geoscience and Remote Sensing Letters, vol. 01, pp. 1-5, 2020.

[16] A. B. Campos, M. I. Pettersson, V. T. Vu, and R. Machado, "False Alarm Reduction in Wavelength-Resolution SAR Change Detection Schemes by Using a Convolutional Neural Network," IEEE Geoscience and Remote Sensing Letters, vol. 01, pp. 1-5, 2020.

[17] Flach, P., "Performance Evaluation in Machine Learning: The Good, the Bad, the Ugly, and the Way Forward," Proceedings of the AAAI Conference on Artificial Intelligence, vol. 33, no. 01, pp.9808-9814.

[18] Ramos, L.P.; Campos, A.B.; Schwartz, C.; Duarte, L.T.; Alves, D.I.; Pettersson, M.I.; Vu, V.T.; Machado, R. A., "Wavelength-Resolution SAR Change Detection Method Based on Image Stack through Robust Principal Component Analysis," Remote Sensing, 2021, 13, 833.

[19] C. Schwartz, L. P. Ramos, L. T. Duarte, M. S. Pinho, M. I. Pettersson, V. T. Vu, R. Machado, "Change Detection in UWB SAR Images Based on Robust Principal Component Analysis," Remote Sensing, 2020, 12, 1916.

[20] A. Pulella, R. S. Aragão, F. Sica, P. Posovszky, P. Rizzoli, "MultiTemporal Sentinel-1 Backscatter and Coherence for Rainforest Mapping," Remote Sensing, 2020, 12, 847.

[21] K. L. de Jong and A. S. Bosman. (2019). Unsupervised Change Detection in Satellite Images Using Convolutional Neural Networks.

[22] R. M. Haralick, K. Shanmugam and I. Dinstein, "Textural Features for Image Classification," IEEE Transactions on Systems, Man, and Cybernetics, vol. SMC-3, no. 06, pp. 610-621, Nov. 1973.

[23] K.I. Laws, "Texture energy measures," Proc. image understanding workshop, (1979), pp. 47-51.

[24] M. Unser, "Sum and Difference Histograms for Texture Classification," IEEE Transactions on Pattern Analysis and Machine Intelligence, vol. PAMI-8, no. 01, pp. 118-125, Jan. 1986.

[25] M . D. Sarker, J. Nichol, H. Iz, B. B. Ahmad, and A. Rahman, "Forest Biomass Estimation Using Texture Measurements of HighResolution Dual-Polarization C-Band SAR Data," IEEE Transactions on Geoscience and Remote Sensing, vol. 51, no. 6, pp. 3371-3384, June 2013. 